\title{
NEW EVIDENCE FOR EFFICIENT COLLISIONLESS HEATING OF ELECTRONS AT THE REVERSE SHOCK OF A YOUNG SUPERNOVA REMNANT
}

\author{
Hiroya Yamaguchi ${ }^{1,2,3}$, Kristoffer A. Eriksen ${ }^{4}$, Carles Badenes ${ }^{5}$, John P. Hughes ${ }^{6}$, Nancy S. Brickhouse ${ }^{3}$, \\ Adam R. Foster ${ }^{3}$, Daniel J. Patnaude ${ }^{3}$, Robert Petre ${ }^{1}$, Patrick O. Slane ${ }^{3}$, and Randall K. Smith ${ }^{3}$ \\ ${ }^{1}$ NASA Goddard Space Flight Center, Code 662, Greenbelt, MD 20771, USA; hiroya.yamaguchi@ nasa.gov \\ ${ }^{2}$ Department of Astronomy, University of Maryland, College Park, MD 20742, USA \\ ${ }^{3}$ Harvard-Smithsonian Center for Astrophysics, 60 Garden Street, Cambridge, MA 02138, USA \\ ${ }^{4}$ Los Alamos National Laboratory, P.O. Box 1663, Los Alamos, NM 87545, USA \\ ${ }^{5}$ Department of Physics and Astronomy and Pittsburgh Particle Physics, Astrophysics and Cosmology Center (PITT PACC), \\ University of Pittsburgh, 3941 O'Hara St, Pittsburgh, PA 15260, USA \\ ${ }^{6}$ Department of Physics and Astronomy, Rutgers University, 136 Frelinghuysen Road, Piscataway, NJ 08854, USA \\ Received 2013 September 6; accepted 2013 November 16; published 2013 December 18
}

\begin{abstract}
Although collisionless shocks are ubiquitous in astrophysics, certain key aspects of them are not well understood. In particular, the process known as collisionless electron heating, whereby electrons are rapidly energized at the shock front, is one of the main open issues in shock physics. Here, we present the first clear evidence for efficient collisionless electron heating at the reverse shock of Tycho's supernova remnant (SNR), revealed by Fe K diagnostics using high-quality X-ray data obtained by the Suzaku satellite. We detect $\mathrm{K} \beta(3 p \rightarrow 1 s)$ fluorescence emission from low-ionization $\mathrm{Fe}$ ejecta excited by energetic thermal electrons at the reverse shock front, which peaks at a smaller radius than $\mathrm{Fe} \mathrm{K} \alpha(2 p \rightarrow 1 s)$ emission dominated by a relatively highly ionized component. Comparisons with our hydrodynamical simulations imply instantaneous electron heating to a temperature 1000 times higher than expected from Coulomb collisions alone. The unique environment of the reverse shock, which is propagating with a high Mach number into rarefied ejecta with a low magnetic field strength, puts strong constraints on the physical mechanism responsible for this heating and favors a cross-shock potential created by charge deflection at the shock front. Our sensitive observation also reveals that the reverse shock radius of this SNR is about $10 \%$ smaller than the previous measurement using the Fe K $\alpha$ morphology from the Chandra observations. Since strong $\mathrm{Fe} \mathrm{K} \beta$ fluorescence is expected only from low-ionization plasma where Fe ions still have many $3 p$ electrons, this feature is key to diagnosing the plasma state and distribution of the immediate postshock ejecta in a young SNR.
\end{abstract}

Key words: atomic data - hydrodynamics - ISM: individual objects (SN 1572, Tycho's SNR) - ISM: supernova remnants - shock waves - X-rays: ISM

Online-only material: color figures

\section{INTRODUCTION}

A supersonic flow colliding with another medium will form a shock wave, which can be described as a discontinuity in the physical conditions of the flow. Shock waves have been extensively observed on Earth and also in a number of astrophysical settings with a wide range of spatial scales: from the solar wind (e.g., Tidman \& Krall 1971; Schwartz et al. 1988) to afterglows of gamma-ray bursts (GRBs; e.g., Meszaros \& Rees 1997; Sari et al. 1998) and merging galaxy clusters (e.g., Markevitch et al. 2005; Markevitch \& Vikhlinin 2007). Unlike terrestrial shocks, the low densities found in some astrophysical environments imply that the shock transition occurs on scales much shorter than the typical particle mean free path for Coulomb collisions. Therefore, collisionless processes involving collective interactions between particles and electromagnetic fields must be responsible for the shock formation (McKee 1974). Despite the ubiquity and importance of these collisionless shocks in astrophysics, the detailed physical processes that determine their fundamental properties are still poorly understood.

One particularly mysterious process, which might be closely related to the formation of the collisionless shock front itself, is the rapid heating of electrons at the shock transition. A strong shock with velocity $v_{s}$ will result in a downstream temperature $T_{i}=3 m_{i} v_{s}^{2} / 16 k_{\mathrm{B}}$, where $m_{i}$ is the mass of particle $i$ and $k_{\mathrm{B}}$ is the Boltzmann constant. Since the timescale for collisional equilibration between different species is much longer than the time a particle spends in the shock transition zone (Spitzer 1962), the equation above can be applied independently to each species $i$, unless collisionless processes contribute to the temperature equilibration. It follows that the electron temperature $\left(T_{e}\right)$ should be much lower than the temperature of heavier ions $\left(T_{\text {ion }}\right)$ immediately behind the shock and they will slowly equilibrate to a common temperature via Coulomb collisions further downstream. However, a number of theoretical investigations have suggested that rapid collisionless electron heating can occur at shock fronts (e.g., McKee 1974; Cargill \& Papadopoulos 1988; Laming 2000; Ghavamian et al. 2007).

Supernova remnants (SNRs) offer an ideal site to study this heating observationally, because they form long-lived, fast shocks in both the interstellar medium (ISM) and the supernova ejecta. To date, most work has concentrated on Balmer-dominated shocks associated with SNR blast waves expanding into the ISM (e.g., Raymond et al. 1983; Laming et al. 1996; Ghavamian et al. 2001, 2007; Rakowski et al. 2003; Helder et al. 2011). Studies of electron heating in reverse shocks (RSs), which are propagating into the supernova ejecta are, on the other hand, very limited (Hamilton et al. 1997; France et al. 2011). In this paper, we focus on the RS of Tycho's SNR, the remnant of the Type Ia supernova observed in A.D. 1572.

There are a number of important differences in environment between blast waves and RSs. Unlike the ISM, supernova ejecta generally have only a small fraction of neutral particles, because the strong ultraviolet/soft X-ray flux from the shocked material 


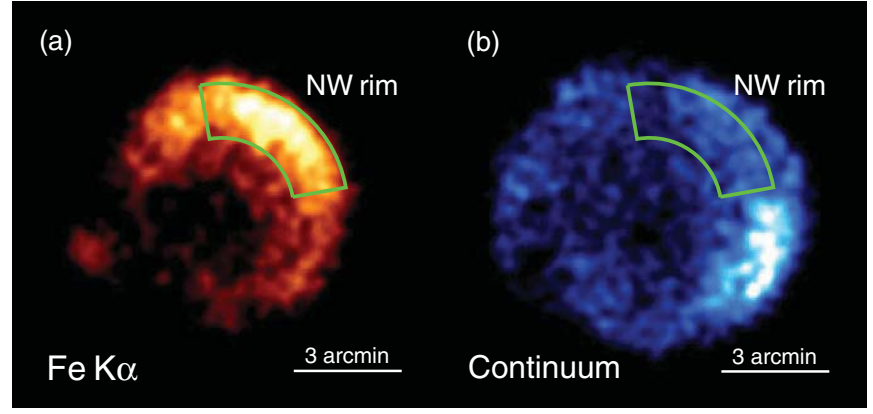

Figure 1. Suzaku XIS images of Tycho's SNR in the (a) 6.43-6.53 keV (Fe K $\alpha$ ) band and (b) 7.7-9.0 keV band (continuum emission). North is up and east is to the left. The northwest (NW) region confined with the green lines is where we extract the spectrum shown in Figures 2 and 4.

(A color version of this figure is available in the online journal.)

can easily photoionize the heavy elements of the unshocked ejecta (e.g., Hamilton et al. 1997). Furthermore, in a Type Ia SNR the ejecta consist only of heavy elements, with no contribution of hydrogen and helium. The magnetic field strength expected in the interior of the white dwarf before the explosion ( $B \lesssim 10^{13} \mathrm{G}$; Suh \& Mathews 2000 ) will be dramatically diluted by expansion. From flux conservation, a conservative upper limit on the field strength present near the RS location in Tycho's SNR is estimated to be $\sim 10^{-7} \mathrm{G}$. This is sufficiently high that the ion gyroradius, $r_{\mathrm{g}} \sim 10^{13}(A / 56)\left(B / 10^{-7}[\mathrm{G}]\right)^{-1} \mathrm{~cm}$ (where $A$ is the mass number), is much smaller than the SNR radius of $\sim 10^{19} \mathrm{~cm}$, ensuring the formation of a collisionless RS. Yet, the inferred strength is at least an order of magnitude below the typical value in the ISM (a few times $10^{-6} \mathrm{G}$ ). The geometry of the interior magnetic field should be highly ordered as the field is stretched radially by the expanding ejecta. These distinct properties, strongly contrasting with those in the ISM, allow us to probe the physics of collisionless electron heating under conditions far different from those of any earlier work.

Previous hydrodynamical calculations applied to Tycho's SNR required the presence of a modest amount of collisionless electron heating at the RS to explain the observed X-ray flux from the shocked ejecta (Badenes et al. 2005, 2006). This is in contrast to earlier results on another Type Ia SNR, SN 1006, where little evidence for collisionless electron heating was found (Hamilton et al. 1997). Since the RS in Tycho's SNR has begun to propagate into the Fe-dominated ejecta (e.g., Hwang et al. 1998; Warren et al. 2005; Badenes et al. 2006), the emission lines from $\mathrm{Fe}$ directly probe the conditions (i.e., electron temperature) in the postshock region. In general, the initial shock-heated material in SNRs is at a very low charge state and only gradually becomes ionized by collisions with hot free electrons. The ejecta in young SNRs are, therefore, in a non-equilibrium ionization (NEI) condition (Masai 1984). Collisional interactions between hot electrons and low-ionized Fe in an NEI plasma produce inner $\mathrm{K}$-shell ionization of the Fe ions, followed by $\mathrm{K} \alpha(2 p \rightarrow 1 s)$ or $\mathrm{K} \beta(3 p \rightarrow 1 s)$ fluorescence transitions (e.g., Palmeri et al. 2003; Mendoza et al. 2004). These emission lines are excellent diagnostics of the temperature and charge population in the shocked material.

Here, we present new, strong evidence for collisionless electron heating at the RS of Tycho's SNR, revealed by Fe K-shell X-ray lines from sensitive observations with the X-ray Imaging Spectrometer (XIS) on board the Suzaku satellite. Our observations allow the first-ever detailed study of weak Fe $\mathrm{K} \beta$ emission alongside the stronger $\mathrm{Fe} \mathrm{K} \alpha$ line. In Section 2, we

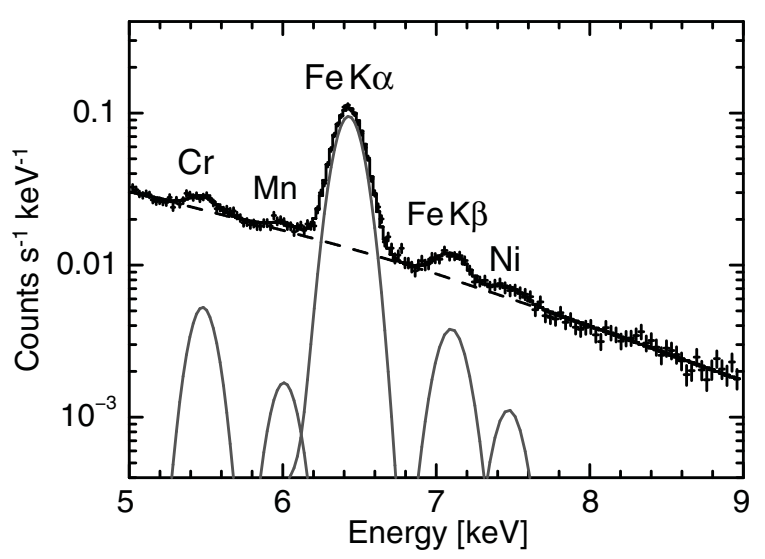

Figure 2. XIS spectrum of Tycho's SNR in the 5.0-9.0 keV band, where the background data are subtracted. A phenomenological model using a power law (dashed line for the continuum) and five Gaussians (solid gray lines for the emission lines) yields the best-fit parameters given in Table 1 .

analyze observational data based on the state-of-the-art atomic physics models we compute. In Section 3, we constrain the efficiency of collisionless electron heating by comparing our hydrodynamical calculations and finally we discuss the plausible mechanism of this efficient heating process.

\section{OBSERVATIONAL RESULTS}

The observations with the Suzaku XIS were performed during 2008 August with a total effective exposure of $415 \mathrm{ks}$. The primary data reduction was performed in accordance with the standard procedure recommended by the instrument team. We use only the data of front-illuminated CCDs (XIS0 and 3) and merge them to improve the photon statistics. Figure 1 shows XIS images in the energy bands of the $\mathrm{Fe} \mathrm{K} \alpha$ line ((a) 6.43-6.53 keV) and continuum emission ((b) 7.7-9.0 keV). This continuum has been shown to be dominated by synchrotron radiation from relativistic electrons accelerated at the blast wave (Hwang et al. 2002; Cassam-Chenaï et al. 2007). We extract an X-ray spectrum from the northwest (NW) rim indicated in Figure 1. Background data are taken from the nearby sky and subtracted from the source spectrum. Figure 2 is the resultant spectrum in the 5.0-9.0 keV band, where emission from $\mathrm{Cr} \mathrm{K} \alpha, \mathrm{Mn} \mathrm{K} \alpha, \mathrm{Fe}$ $\mathrm{K} \alpha$ and $\mathrm{K} \beta$, and $\mathrm{Ni} \mathrm{K} \alpha$ is clearly resolved. We note that this is the first detection of $\mathrm{Ni}$ from this remnant, although we do not focus on this element in this paper.

\subsection{Fe K Emission Diagnostics}

We measure the centroid energies of the $\mathrm{Fe} \mathrm{K} \alpha\left(E_{\mathrm{K} \alpha}\right)$ and $\mathrm{K} \beta\left(E_{\mathrm{K} \beta}\right)$ line blends and their flux ratio $(\mathcal{R})$ using Gaussian functions and obtain $E_{\mathrm{K} \alpha}=6435 \pm 1( \pm 6) \mathrm{eV}, E_{\mathrm{K} \beta}=$ $7104 \pm 10( \pm 7) \mathrm{eV}$, and $\mathcal{R}=5.5_{-0.5}^{+0.6} \%$. The parenthetical values are the instrumental systematic uncertainty in the energy scale, $0.1 \%$ of the mean energy, due to the incomplete gain calibration of the XIS (Ozawa et al. 2009). The continuum is simply modeled by a power law, giving a photon index of $\Gamma \sim 2.9$. The best-fit parameters for all the emission lines are given in Table 1. During the analysis, an absorption column density of $N_{\mathrm{H}}=7 \times 10^{21} \mathrm{~cm}^{-2}$ (Cassam-Chenaï et al. 2007) with a solar elemental composition (Wilms et al. 2000) was assumed, although the analyzed energy band was not affected by the foreground extinction. To evaluate the systematic uncertainty due to background subtraction, we perform fits using a different background data set that includes only the instrumental component 


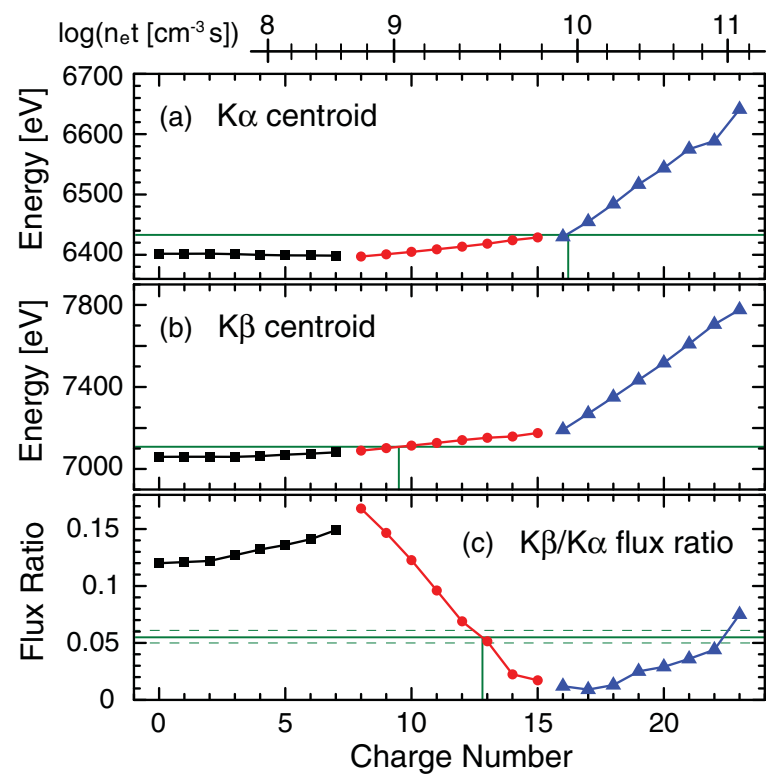

Figure 3. Expected centroid energies of the (a) $\mathrm{Fe} \mathrm{K} \alpha$ and (b) $\mathrm{Fe} \mathrm{K} \beta$ emission, and (c) the $\mathrm{K} \beta / \mathrm{K} \alpha$ flux ratio as a function of the charge number $z$ of Fe ions, with the corresponding ionization ages $\left(n_{e} t\right)$ indicated at the top. The best-fit values for Tycho's SNR are shown with the solid green lines. The dashed green lines in panel (c) indicate the $1 \sigma$ lower and upper limits of the observed value. The black squares and red circles are the values calculated using the atomic data of Palmeri et al. (2003) and Mendoza et al. (2004), respectively. We also use the FAC to calculate the rate coefficients of collisional ionization and excitation. The blue triangles are obtained by full calculations using the FAC (K. A. Eriksen et al., in preparation).

(A color version of this figure is available in the online journal.)

Table 1

Best-fit Spectral Parameters for the NW Rim

\begin{tabular}{lccc}
\hline \hline Emission & $\begin{array}{c}\text { Centroid } \\
(\mathrm{eV})\end{array}$ & $\begin{array}{c}\text { FWHM } \\
(\mathrm{eV})\end{array}$ & $\begin{array}{c}\text { Flux } \\
\left(10^{-6} \text { photons cm }^{-2} \mathrm{~s}^{-1}\right)\end{array}$ \\
\hline $\mathrm{Cr} \mathrm{K} \alpha$ & $5482_{-12}^{+10}$ & $141_{-45}^{+35}$ & $5.05_{-0.69}^{+0.72}$ \\
$\mathrm{Mn} \mathrm{K} \alpha$ & $6012_{-26}^{+25}$ & 141 & $1.73_{-0.46}^{+0.47}$ \\
$\mathrm{Fe} \mathrm{K} \alpha$ & $6435 \pm 1$ & $138 \pm 2$ & $107 \pm 1$ \\
$\mathrm{Fe} \mathrm{K} \beta$ & $7104 \pm 10$ & $160 \pm 42$ & $5.62_{-0.56}^{+0.61}$ \\
$\mathrm{Ni} \mathrm{K} \alpha$ & $7478 \pm 32$ & 138 & $1.82_{-0.43}^{+0.42}$ \\
\hline
\end{tabular}

Notes. The uncertainties are the statistical component in the $1 \sigma$ confidence range. The Gaussian widths (FWHM) of the Mn K $\alpha$ and Ni $\mathrm{K} \alpha$ lines are linked to those of $\mathrm{Cr} \mathrm{K} \alpha$ and $\mathrm{Fe} \mathrm{K} \alpha$, respectively.

(non-X-ray background). No significant change is found in the measurement of the line centroids and intensities.

The observed Fe-line parameters, $E_{\mathrm{K} \alpha}, E_{\mathrm{K} \beta}$, and $\mathcal{R}$, are compared in Figure 3 with the theoretically expected values for different charge numbers $z$ (where $z=1$ indicates singly ionized Fe), which are also tabulated in Table 2. For $z=0-7$ and $z=8-16$, we use level energies, Einstein $A$-values, and fluorescence yields provided in the archival database of Palmeri et al. (2003) and Mendoza et al. (2004), respectively. Since the transition probabilities of forbidden processes (e.g., $2 s \rightarrow 1 s$ ) are negligible in multiple-electron ions (Palmeri et al. 2003; Mendoza et al. 2004), we take into account only $2 p \rightarrow 1 s$ and $3 p \rightarrow 1 s$ transitions as radiation channels for the $\mathrm{K} \alpha$ and $\mathrm{K} \beta$ emission. We calculate rate coefficients for collisional ionization and excitation for each charge number using the "Flexible Atomic Code" (FAC; Gu 2008). For $z \geqslant 16$, we perform full computational calculations with the FAC to obtain
Table 2

Theoretical Values of the $\mathrm{Fe} \mathrm{K} \alpha$ and $\mathrm{K} \beta$ Centroid Energies and their Intensity Ratios for the Different Charge Numbers $z$

\begin{tabular}{lccccccc}
\hline \hline$z$ & $\begin{array}{c}E_{\mathrm{K} \alpha} \\
(\mathrm{eV})\end{array}$ & $\begin{array}{c}E_{\mathrm{K} \beta} \\
(\mathrm{eV})\end{array}$ & $\mathcal{R}$ & $z$ & $\begin{array}{c}E_{\mathrm{K} \alpha} \\
(\mathrm{eV})\end{array}$ & $\begin{array}{c}E_{\mathrm{K} \beta} \\
(\mathrm{eV})\end{array}$ & $\mathcal{R}$ \\
\hline 0 & 6402 & 7059 & 0.120 & 12 & 6414 & 7141 & 0.069 \\
1 & 6402 & 7060 & 0.121 & 13 & 6419 & 7153 & 0.052 \\
2 & 6402 & 7060 & 0.122 & 14 & 6425 & 7159 & 0.022 \\
3 & 6401 & 7059 & 0.127 & 15 & 6428 & 7176 & 0.010 \\
4 & 6400 & 7063 & 0.132 & 16 & 6427 & 7192 & 0.012 \\
5 & 6399 & 7070 & 0.136 & 17 & 6455 & 7270 & 0.009 \\
6 & 6399 & 7075 & 0.141 & 18 & 6484 & 7351 & 0.013 \\
7 & 6399 & 7081 & 0.149 & 19 & 6517 & 7434 & 0.025 \\
8 & 6398 & 7090 & 0.168 & 20 & 6544 & 7517 & 0.029 \\
9 & 6401 & 7102 & 0.146 & 21 & 6575 & 7610 & 0.036 \\
10 & 6405 & 7115 & 0.122 & 22 & 6589 & 7705 & 0.044 \\
11 & 6410 & 7128 & 0.096 & 23 & 6641 & 7777 & 0.075 \\
\hline
\end{tabular}

the theoretical values (K. A. Eriksen et al., in preparation). During the calculations, we assume an electron temperature of $5 \mathrm{keV}$. The temperature dependence is found to be significant only for $z \geqslant 20$, where the inner K-shell excitation rate becomes dominant over the K-shell ionization rate. Since the population of such highly charged Fe is not substantial in Tycho's SNR, as discussed below, the diagnostics we perform here are essentially independent of the electron temperature of the Fe ejecta.

As found in Figure 3(a), the observed $E_{\mathrm{K} \alpha}$ value corresponds to the charge states $\mathrm{Fe}^{15+} \sim \mathrm{Fe}^{17+}$ and an ionization age $\left(n_{e} t\right)$ of $\sim 1 \times 10^{10} \mathrm{~cm}^{-3} \mathrm{~s}$, where $n_{e}$ and $t$ are the electron density and the time elapsed since shock heating, respectively. This result is consistent with several previous measurements (e.g., Hwang et al. 1998; Hayato et al. 2010). It has, therefore, frequently been assumed that all the shocked ejecta responsible for the $\mathrm{Fe} \mathrm{K}$-shell emission have this ionization age. We find in Figure 3(b), however, that the observed $E_{\mathrm{K} \beta}$ value corresponds to significantly lower charge states $\mathrm{Fe}^{8+} \sim \mathrm{Fe}^{10+}$, with an ionization age of $\sim 1 \times 10^{9} \mathrm{~cm}^{-3} \mathrm{~s}$, about 10 times lower than that indicated by the Fe $\mathrm{K} \alpha$ centroid. The $\mathrm{K} \beta$-to-K $\alpha$ flux ratio $(\mathcal{R})$ is also sensitive to the charge number, especially in the range $z=8-14$ (Figure 3(c)). In this regime, the flux ratio experiences a drastic decrease because the Fe ions lose their $3 p$ shell electrons (which are responsible for the $\mathrm{K} \beta$ fluorescence) as $z$ increases. The observed value is closest to the expected ratio for $z=13$, intermediate between the results from the $\mathrm{K} \alpha$ and $\mathrm{K} \beta$ centroids.

The inconsistency among the three diagnostics indicates the presence of a range of plasma conditions, with the $\mathrm{K} \alpha$ emission being dominated by more highly ionized $\mathrm{Fe}$ and the $\mathrm{K} \beta$ emission by less ionized Fe. We re-fit the NW spectrum applying a 'two-component' model for the Fe emission. The red Gaussians in Figure 4 correspond to the higher ionization component, where $E_{\mathrm{K} \beta}$ and $\mathcal{R}$ are fixed to $7200 \mathrm{eV}$ and $1 \%$ (the values theoretically expected for $z \sim 16)$. Only the $\mathrm{K} \alpha$ centroid is allowed to vary, yielding $E_{\mathrm{K} \alpha}=6447_{-3}^{+2} \mathrm{eV}$, which is in between the values for $\mathrm{Fe}^{16+}$ and $\mathrm{Fe}^{17+}$. The contribution of the lower ionization component is indicated by the green Gaussians in Figure 4, where we fix $E_{\mathrm{K} \alpha}$ and $\mathcal{R}$ to $6400 \mathrm{eV}$ and $15 \%$ (as expected for $z \sim 8)$. The best-fit $E_{\mathrm{K} \beta}$ value of $7090 \pm 11 \mathrm{eV}$ is consistent with that for $\mathrm{Fe}^{8+}$. To summarize, we are able to explain all the $\mathrm{Fe}$ K-shell emission self-consistently with this simple twocomponent model using different ionization states and the expected $\mathrm{K} \beta / \mathrm{K} \alpha$ emissivity ratios. 


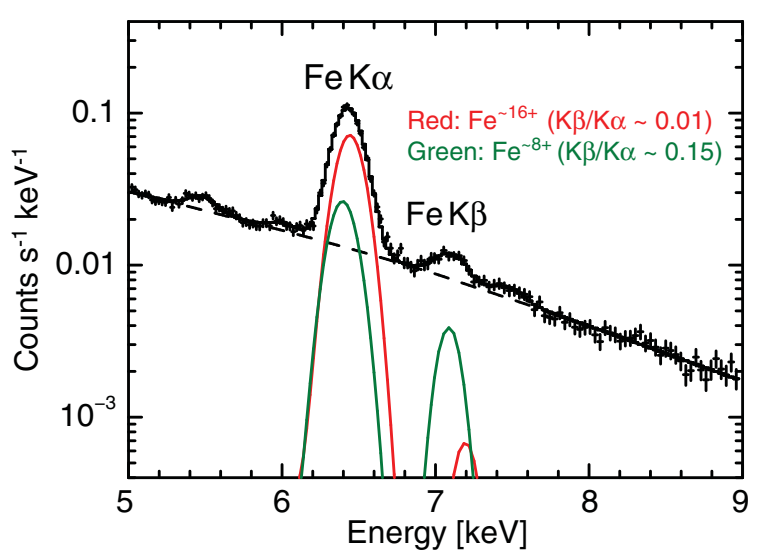

Figure 4. Same spectral data as in Figure 2, but the "two-component" model is applied to the $\mathrm{Fe} \mathrm{K}$ emission (see the text for details). Red and green represent the higher-ionization (around $\mathrm{Fe}^{16+}$ ) and lower-ionization (around $\mathrm{Fe}^{8+}$ ) components, respectively. The $\mathrm{K} \beta / \mathrm{K} \alpha$ flux ratio is expected to be lower in the former $(\sim 0.01)$ than the latter $(\sim 0.15)$, because $\mathrm{Fe}^{16+}$ ions have no bound electron in the $3 p$ shell, which is responsible for the $\mathrm{K} \beta$ emission.

(A color version of this figure is available in the online journal.)

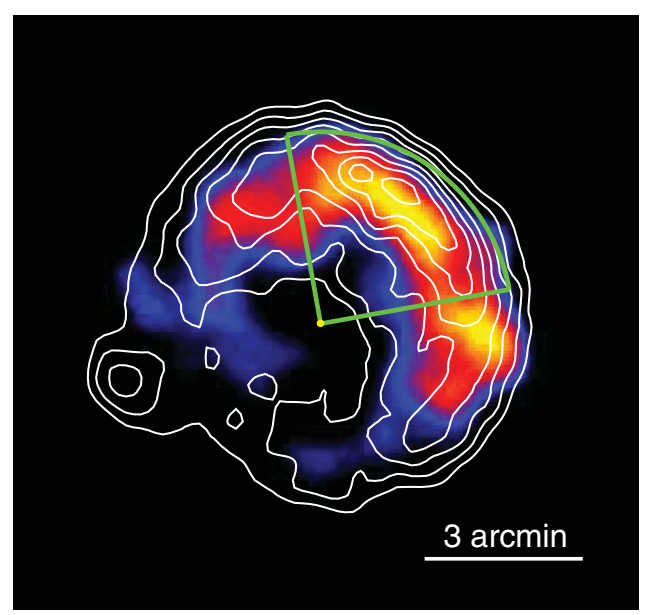

Figure 5. XIS image of Tycho's SNR in the 7.0-7.2 $\mathrm{keV}(\mathrm{Fe} \mathrm{K} \beta$ ) band, where the $\mathrm{Fe} \mathrm{K} \alpha$ image (same as Figure 1(a)) is overplotted in contours. The synchrotron continuum flux estimated using Figure 1(b) is subtracted from the raw $\mathrm{Fe} \mathrm{K} \beta$ image. The morphology is subject to some uncertainties since a spatially uniform photon index is assumed in this subtraction procedure. It is nevertheless clear that the $\mathrm{Fe} \mathrm{K} \beta$ emission peaks at a smaller radius than the $\mathrm{K} \alpha$ emission in the bright NW rim. The green sector indicates where the spatially resolved spectral analysis (Figure 6) is performed.

(A color version of this figure is available in the online journal.)

\subsection{Spatial Analysis}

The interpretation in the previous subsection predicts that the Fe $\mathrm{K} \beta$ emission peaks interior to the $\mathrm{K} \alpha$ emission, because the innermost ejecta were heated by the RS more recently and so should have a lower ionization age than the outer ejecta. We perform a spatial analysis to verify that this is indeed the case. Since the $\mathrm{Fe} \mathrm{K} \beta$ emission is not as strong as the synchrotron continuum flux in the same energy band, subtraction of the continuum component is necessary. We estimate the continuum level in the 7.0-7.2 $\mathrm{keV}(\mathrm{Fe} \mathrm{K} \beta$ ) band by scaling the synchrotron $\mathrm{X}$-ray image (Figure 1(b)) using a photon index of 2.9 (the best-fit value for the NW rim spectrum). The color image in Figure 5 is created by subtracting these scaled data from the raw 7.0-7.2-keV image, where contours of the $\mathrm{K} \alpha$ emission (corresponding to the image in Figure 1(a)) are overlaid. As we expected, the $\mathrm{K} \beta$ emission has a smaller peak radius than the

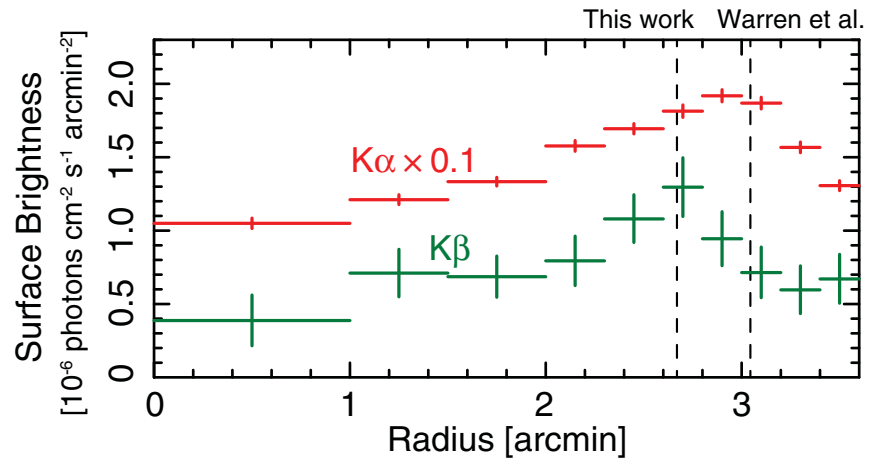

Figure 6. Radial profiles for the surface brightness of the $\mathrm{Fe} \mathrm{K} \alpha$ and $\mathrm{K} \beta$ emission in the NW quadrant, derived from the spatially resolved spectral analysis. The smaller peak radius of the $\mathrm{Fe} \mathrm{K} \beta$ is confirmed. The RS positions determined by Warren et al. (2005) and this work are indicated with the dashed lines.

(A color version of this figure is available in the online journal.)

other. It should be noted, however, that this imaging analysis has some uncertainties, because we assume that the photon index of the continuum X-rays is spatially uniform over the entire region. This is actually not the case, but the synchrotron emission in Tycho's SNR is known to have spatially inhomogeneous hardness (Cassam-Chenaï et al. 2007; Eriksen et al. 2011).

This motivates our spatially resolved spectral analysis, which gives a more quantitative measurement of the $\mathrm{Fe} \mathrm{K} \beta$ distribution. The azimuthal sector shown in Figure 5 is divided into 10 radial zones of variable width (to account for the variation in brightness) for spectral extraction, by assuming the same center position for the SNR as that determined by the previous Chandra observations (Warren et al. 2005). The spectrum from each region is fit with the same model applied to the NW rim (five Gaussians plus a power law), allowing the photon index as well as the other parameters to vary freely. The resulting surface brightness profiles of the $\mathrm{Fe} \mathrm{K} \alpha$ and $\mathrm{K} \beta$ emission are shown in Figure 6, confirming the smaller peak radius of the $\mathrm{K} \beta$ emission, while the $\mathrm{K} \alpha$ peak radius is consistent with that from the Chandra data.

Our analysis demonstrates that the radial profile of the $\mathrm{Fe} \mathrm{K} \beta$ emission is more sensitive to the immediate postshock ejecta than the $\mathrm{K} \alpha$ emission, revealing a $\sim 10 \%$ smaller $\mathrm{RS}$ radius than was previously determined using the Fe $\mathrm{K} \alpha$ morphology from the Chandra observations (Warren et al. 2005). Assuming a simple shell geometry, which predicts that the maximum surface brightness will coincide with the inner edge of the shell (Warren et al. 2005), we estimate the RS radius to be $7.1 \times 10^{18}(D / 3.0[\mathrm{kpc}]) \mathrm{cm}$, or $2.3(D / 3.0[\mathrm{kpc}]) \mathrm{pc}$, where $D$ is the distance to Tycho's SNR. This is about $63 \%$ of the SNR blast wave radius, $3.6(D / 3.0[\mathrm{kpc}]) \mathrm{pc}$.

\section{INTERPRETATION}

\subsection{Comparison with Hydrodynamical Calculations}

Our new X-ray measurements have revealed that $\mathrm{Fe}$ in the innermost ejecta is in an extremely low ionization state $(z \lesssim 8)$. Yet, the strong $\mathrm{Fe} \mathrm{K} \beta$ emission requires the electron temperature near the RS front be high enough to ionize the inner K-shell electrons of these low- $z$ ions. Keeping this in mind, we constrain the efficiency of collisionless electron heating by comparing our results with one-dimensional hydrodynamical simulations that incorporate an NEI calculation (Badenes et al. 2006). For the initial conditions, we assume the ejecta structure expected for a typical delayed-detonation Type Ia supernova with an $\mathrm{Fe}$ 


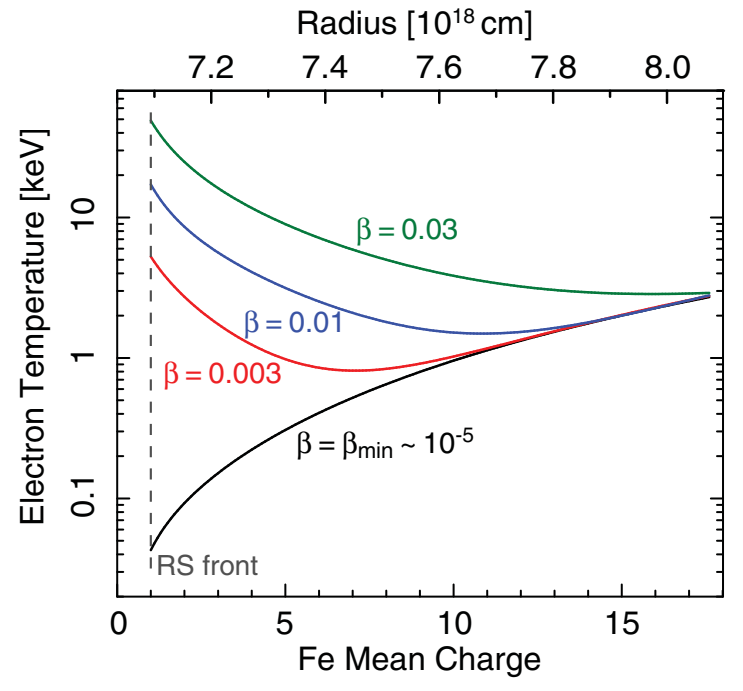

Figure 7. Electron temperature as a function of the mean charge of Fe ions from our hydrodynamical simulations. The corresponding radius is also given above. The black curve is the $\beta_{\min }$ model where no collisionless electron heating is assumed. The temperature ratio between the electrons and ions at the RS front is, therefore, set by their mass ratio. The models represented by the red, blue, and green curves assume that collisionless electron heating occurs at the $\mathrm{RS}$, parameterized by ( $\beta=T_{e} / T_{\text {ion }}$ ) with values set to $0.003,0.01$, and 0.03 , respectively.

(A color version of this figure is available in the online journal.)

yield of $\sim 0.8 M_{\odot}$ and an explosion energy of $\sim 1.2 \times 10^{51} \mathrm{erg}$. This model reproduces the fundamental properties of Tycho's supernova: the historical light curve (Ruiz-Lapuente 2004) and modern light echo spectrum (Rest et al. 2008; Krause et al. 2008). The SNR evolution is followed to the age of Tycho's SNR assuming a uniform ambient density $\left(\rho_{\mathrm{AM}}\right)$ of $2 \times 10^{-24} \mathrm{~g} \mathrm{~cm}^{-3}$. The result is also in good agreement with the basic dynamics (e.g., angular size, shock speed) of the SNR for a reasonable value of the distance (Badenes et al. 2006). The effect of collisionless electron heating is introduced to our NEI calculations by parameterizing the electron-to-ion temperature ratio just behind the $\mathrm{RS}, \beta=T_{e} / T_{\text {ion }}$.

Figure 7 shows the relationship among charge state, radius, and electron temperature for several values of $\beta$. The black curve is derived under the assumption that the initial temperature of each species follows the equation $T_{i}=3 m_{i} v_{s}^{2} / 16 k_{\mathrm{B}}$, which leads to $T_{e} / T_{\mathrm{Fe}}=m_{e} / m_{\mathrm{Fe}} \sim 10^{-5}$ at the RS front (hereafter, the $\beta_{\min }$ model). The other models, shown as the red, blue, and green curves, have larger values of $\beta$, which result in higher electron temperatures in the postshock region (hereafter, the collisionless heating models). Subsequent temperature changes are due to collisional processes, heating via ion-electron Coulomb collisions (dominant in the $\beta_{\text {min }}$ model) and cooling via the ongoing collisional ionization process (prominent in the collisionless heating models). The $\beta_{\text {min }}$ model predicts an electron temperature of $\lesssim 1 \mathrm{keV}$ in the region dominated by $z \leqslant 10$ $\mathrm{Fe}$ ions. The free electrons in this region are, therefore, not energetic enough to produce significant K-shell ionization and subsequent fluorescence. This is shown more quantitatively in Figure 8(a), where we plot the expected $\mathrm{Fe} \mathrm{K} \alpha$ and $\mathrm{K} \beta$ luminosity for each charge state. The $\beta_{\min }$ model clearly fails to reproduce the strong $\mathrm{K} \beta$ emission from low-ionized $\mathrm{Fe}$, in direct conflict with our observations. In contrast, the collisionless heating models can reproduce the emission from the broad $\mathrm{Fe}$ ion population, as illustrated in Figure 8(b) (corresponding to $\beta=0.01$ ).

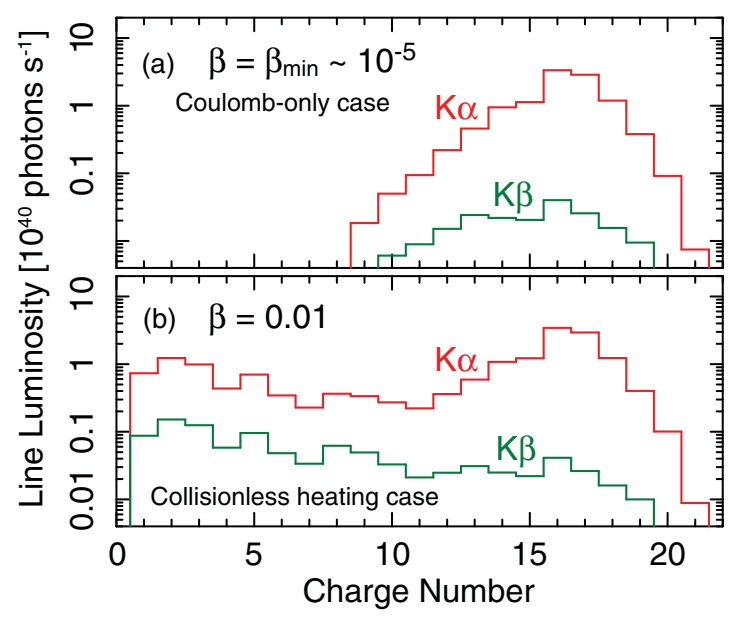

Figure 8. (a) Predicted luminosities of the $\mathrm{Fe} \mathrm{K} \alpha$ (red) and $\mathrm{K} \beta$ (green) emission lines for the $\beta_{\min }$ model. Owing to the low electron temperature in the innermost region, little or no emission from low-ionized $(z<10)$ Fe is expected. (b) Same as panel (a), but for the model with $\beta=0.01$. Emission from $\mathrm{Fe}$ with various charge states, including $z<10$ where higher $\mathcal{R}$ values are achieved, is expected. (A color version of this figure is available in the online journal.)

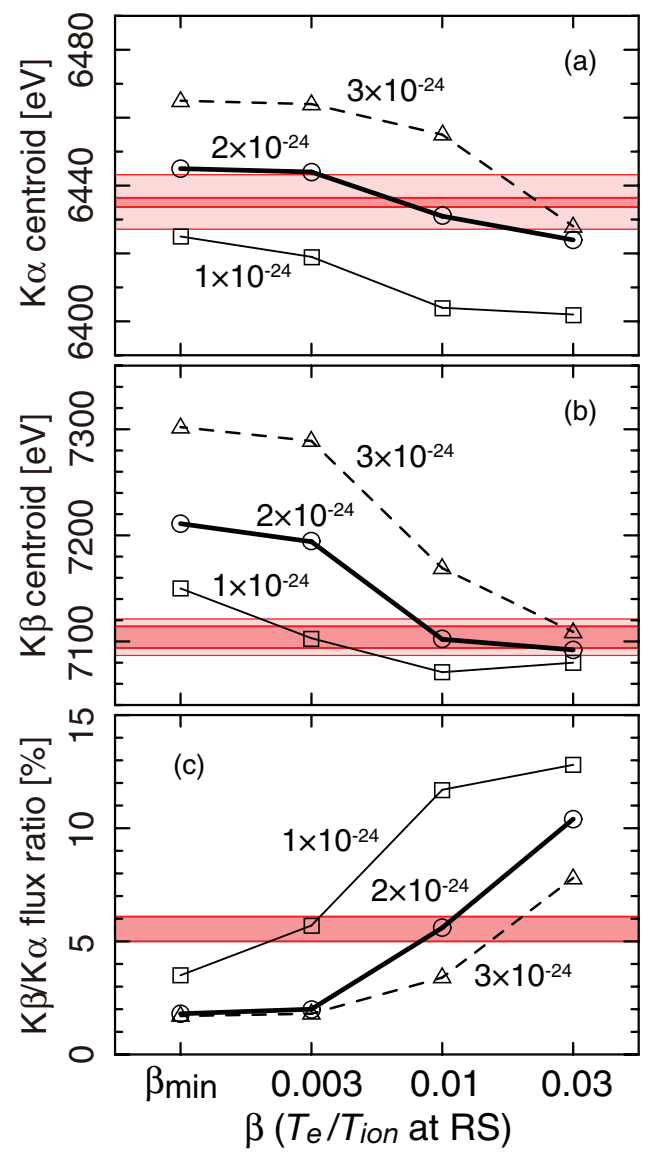

Figure 9. Comparison between the predicted values for (a) $E_{\mathrm{K} \alpha}$, (b) $E_{\mathrm{K} \beta}$, and (c) $\mathcal{R}$ and the observed values, for which statistical and systematic uncertainties are indicated with dark and light red regions, respectively. The thin solid (with squares), thick solid (with circles), and dashed (with triangles) lines correspond to ambient densities $\left(\rho_{\mathrm{AM}}\right)$ of $1 \times 10^{-24}, 2 \times 10^{-24}$, and $3 \times 10^{-24} \mathrm{~g} \mathrm{~cm}^{-3}$, respectively. We find that the model with $\rho_{\mathrm{AM}}=2 \times 10^{-24} \mathrm{~g} \mathrm{~cm}^{-3}$ and $\beta=0.01$ provides the best match to the observations.

(A color version of this figure is available in the online journal.)

In Figure 9, we compare the model-predicted values of $E_{\mathrm{K} \alpha}$, $E_{\mathrm{K} \beta}$, and $\mathcal{R}$ with the observed values for Tycho's SNR, where the impact of different ambient density values, $\rho_{\mathrm{AM}}=1 \times 10^{-24}$ 
and $3 \times 10^{-24} \mathrm{~g} \mathrm{~cm}^{-3}$, are also explored. We confirm that the $\beta_{\text {min }}$ models cannot reproduce the low $E_{\mathrm{K} \beta}$ and high $\mathcal{R}$ values we observe for any value of the ambient density. Although a lower $\rho_{\text {AM }}$ value allows for a larger population of low-ionized $\mathrm{Fe}$, it also leads to a $\mathrm{Fe} \mathrm{K} \alpha$ centroid energy far lower than the observed value. Only the collisionless heating model with $\beta=0.01$ and $\rho_{\mathrm{AM}}=2 \times 10^{-24} \mathrm{~g} \mathrm{~cm}^{-3}$ explains all the observed values within the given uncertainties, indicating that when the electrons pass through the RS front, they gain an internal energy about $10^{3}$ times higher than expected without collisionless heating.

It should be noted that the recent infrared observation with Spitzer determined the mean ISM density around Tycho's SNR to be (2-4) $\times 10^{-25} \mathrm{~g} \mathrm{~cm}^{-3}$ (Williams et al. 2013). This is consistent with the previous estimates from the blast wave expansion rate (Katsuda et al. 2010) and the upper limit of thermal X-ray emission from the shocked ISM (Cassam-Chenaii et al. 2007), but is significantly lower than the value from our diagnostics. This discrepancy implies the presence of a density gradient around the progenitor; the SNR blast wave had initially interacted with relatively high-density matter, which enhanced the ionization age of the outer ejecta but is now expanding into the low-density ISM. A similar interpretation was given by Dwarkadas \& Chevalier (1998) and Chiotellis et al. (2013). Although the effect of such a non-uniform ISM should be involved in our future calculations, we believe that this does not affect our conclusion significantly. The Fe K emission from the broad range of ionization ages can be achieved only when the collisionless heating efficiency is high enough, as demonstrated in Figures 8 and 9.

\subsection{Origin of Collisionless Electron Heating}

We have presented the first clear evidence for efficient collisionless heating of electrons at the RS of Tycho's SNR, which is propagating into metal-rich (no-hydrogen) ejecta at low magnetic field strength, with a velocity of $\sim 4000 \mathrm{~km} \mathrm{~s}^{-1}$ (Badenes et al. 2006; Hayato et al. 2010). As the unshocked ejecta in young Type Ia SNRs has a low temperature $(\sim 5000 \mathrm{~K}$ : Hamilton \& Fesen 1988), the Mach number is estimated to be a few thousand. Previously, the nature of collisionless heating at SNR blast waves has been studied via optical spectroscopy of Balmer-dominated shocks (e.g., Raymond et al. 1983; Laming et al. 1996; Ghavamian et al. 2001, 2007; Rakowski et al. 2003; Helder et al. 2011). However, temperature measurements using optical spectra have been subject to large uncertainties in atomic cross sections, especially for high-velocity shocks $\left(v_{s} \gtrsim 1000 \mathrm{~km} \mathrm{~s}^{-1}\right.$; e.g., Heng 2010).

For Balmer-dominated shocks, two main scenarios are suggested as the origin of the electron heating: (1) lower hybrid wave heating in a cosmic-ray precursor (e.g., Laming 2000; Ghavamian et al. 2007) and (2) plasma wave heating due to Buneman instabilities formed by reflected non-Maxwellian ions (e.g., Cargill \& Papadopoulos 1988; Matsukiyo 2010). Both scenarios require a quasi-perpendicular shock, which is unlikely for RSs, where the magnetic field is expected to be quasi-parallel to the fluid flow due to expansion-induced stretch of the field lines. Moreover, there is little evidence for relativistic cosmic rays at the RS of Tycho's SNR (Warren et al. 2005), which makes scenario (a) unlikely in this context. An alternative scenario, the cross-shock potential (e.g., Balikhin et al. 1993), which has also been suggested as the origin of energetic electrons in GRB afterglows (Gedalin et al. 2008; Sironi \& Spitkovsky 2011), may apply to our case. In this model, charge separation is created at the shock front due to the different gyroradii of ions and electrons, forming a potential gap where electrons arriving later can be accelerated. This requires no specific orientation of the background magnetic field and predicts the self-generation of small-scale electromagnetic fields (Gedalin et al. 2008). The high Mach number for the RS also supports this analogy with GRB shocks. Our observation of efficient collisionless heating in the unique environment of a SNR RS suggests that these shocks may be fundamentally different from the more widely studied Balmer-dominated shocks into ISM material.

We are thankful to Drs. John D. Raymond and Timothy R. Kallman for useful information and discussion. This work is supported by funding from NASA Suzaku GO grant NNX08AZ86G (J.P.H.) and NASA ADP grant NNX12AF44G (R.K.S.).

\section{REFERENCES}

Badenes, C., Borkowski, K. J., \& Bravo, E. 2005, ApJ, 624, 198

Badenes, C., Borkowski, K. J., Hughes, J. P., Hwang, U., \& Bravo, E. 2006, ApJ, 645,1373

Balikhin, M., Gedalin, M., \& Petrukovich, A. 1993, PhRvL, 70, 1259

Cargill, P. J., \& Papadopoulos, K. 1988, ApJL, 329, L29

Cassam-Chenaï, G., Hughes, J. P., Ballet, J., \& Decourchelle, A. 2007, ApJ, 665,315

Chiotellis, A., Kosenko, D., Schure, K. M., Vink, J., \& Kaastra, J. S. 2013, MNRAS, 435, 1659

Dwarkadas, V. V., \& Chevalier, R. A. 1998, ApJ, 497, 807

Eriksen, K. A., Hughes, J. P., Badenes, C., et al. 2011, ApJL, 728, L28

France, K., McCray, R., Penton, S. V., et al. 2011, ApJ, 743, 186

Gedalin, M., Balikhin, M. A., \& Eichler, D. 2008, PhRvE, 77, 026403

Ghavamian, P., Laming, J. M., \& Rakowski, C. E. 2007, ApJL, 654, L69

Ghavamian, P., Raymond, J., Smith, R. C., \& Hartigan, P. 2001, ApJ, 547,995

Gu, M. F. 2008, CaJPh, 86, 675

Hamilton, A. J. S., \& Fesen, R. A. 1988, ApJ, 327, 178

Hamilton, A. J. S., Fesen, R. A., Wu, C.-C., Crenshaw, D. M., \& Sarazin, C. L. 1997, ApJ, 481, 838

Hayato, A., Yamaguchi, H., Tamagawa, T., et al. 2010, ApJ, 725, 894

Helder, E. A., Vink, J., \& Bassa, C. G. 2011, ApJ, 737, 85

Heng, K. 2010, PASA, 27, 23

Hwang, U., Decourchelle, A., Holt, S. S., \& Petre, R. 2002, ApJ, 581, 1101

Hwang, U., Hughes, J. P., \& Petre, R. 1998, ApJ, 497, 833

Katsuda, S., Petre, R., Hughes, J. P., et al. 2010, ApJ, 709, 1387

Krause, O., Tanaka, M., Usuda, T., et al. 2008, Natur, 456, 617

Laming, J. M. 2000, ApJS, 127, 409

Laming, J. M., Raymond, J. C., McLaughlin, B. M., \& Blair, W. P. 1996, ApJ, 472, 267

Markevitch, M., Govoni, F., Brunetti, G., \& Jerius, D. 2005, ApJ, 627, 733

Markevitch, M., \& Vikhlinin, A. 2007, PhR, 443, 1

Masai, K. 1984, Ap\&SS, 98, 367

Matsukiyo, S. 2010, PhPl, 17, 042901

McKee, C. F. 1974, ApJ, 188, 335

Mendoza, C., Kallman, T. R., Bautista, M. A., \& Palmeri, P. 2004, A\&A, 414, 377

Meszaros, P., \& Rees, M. J. 1997, ApJ, 476, 232

Ozawa, M., Uchiyama, H., Matsumoto, H., et al. 2009, PASJ, 61, 1

Palmeri, P., Mendoza, C., Kallman, T. R., Bautista, M. A., \& Meléndez, M. 2003, A\&A, 410, 359

Rakowski, C. E., Ghavamian, P., \& Hughes, J. P. 2003, ApJ, 590, 846

Raymond, J. C., Blair, W. P., Fesen, R. A., \& Gull, T. R. 1983, ApJ, 275, 636

Rest, A., Welch, D. L., Suntzeff, N. B., et al. 2008, ApJL, 681, L81

Ruiz-Lapuente, P. 2004, ApJ, 612, 357

Sari, R., Piran, T., \& Narayan, R. 1998, ApJL, 497, L17

Schwartz, S. J., Thomsen, M. F., Bame, S. J., \& Stansberry, J. 1988, JGR, 93, 12923

Sironi, L., \& Spitkovsky, A. 2011, ApJ, 726, 75

Spitzer, L. 1962, Physics of Fully Ionized Gases (New York: Interscience)

Suh, I.-S., \& Mathews, G. J. 2000, ApJ, 530, 949

Tidman, D. A., \& Krall, N. A. 1971, Shock Waves in Collisionless Plasmas (New York: Interscience)

Warren, J. S., Hughes, J. P., Badenes, C., et al. 2005, ApJ, 634, 376

Williams, B. J., Borkowski, K. J., Ghavamian, P., et al. 2013, ApJ, 770, 129

Wilms, J., Allen, A., \& McCray, R. 2000, ApJ, 542, 914 